\title{
Zum Zeeman-Effekt im Rotationsspektrum von Molekülen mit interner Rotation
}

\author{
D. Sutter und A. GuARnieri
}

Abteilung Chemische Physik im Institut für Physikalische Chemie der Universität Kiel

(Z. Naturforsch. 25 a, 1036-] 043 [1970] ; eingegangen am 13. April 1970)

The Hamiltonian for a molecule with internal rotation, rotating in an external magnetic field is derived. Spin effects are neglected.

Die Untersuchung der Zeeman-Aufspaltung im Rotationsspektrum frei rotierender Moleküle erlaubt, kombiniert mit der Messung der Substanzsuszeptibilität, die experimentelle Bestimmung der Diagonalelemente des magnetischen Suszeptibilitätstensors und des $g$-Tensors des betreffenden Moleküls ${ }^{1,2}$.

$$
\begin{gathered}
g_{a a}=\frac{M_{\mathrm{p}}}{I_{a a}^{(n)}}\left\{\sum_{n} Z_{n}\left(b_{n}{ }^{2}+c_{n}{ }^{2}\right)+\frac{2}{m} \sum_{\nu>0} \frac{\left|\left\langle 0\left|L_{a}\right| \nu\right\rangle\right|^{2}}{E_{0}-E_{\nu}}\right\} \\
\chi_{a a}=-\frac{e^{2}}{4 m c^{2}}\left\{\left\langle 0\left|\sum_{e}\left(b_{e}{ }^{2}+c_{e}{ }^{2}\right)\right| 0\right\rangle-\right. \\
\left.\quad-\frac{2}{m} \sum_{\nu>0} \frac{\left|\left\langle 0\left|L_{a}\right| \nu\right\rangle\right|^{2}}{E_{0}-E_{v}}\right\}
\end{gathered}
$$

und zyklisch.

Hierin sind: $M_{\mathrm{p}}=$ Protonenmasse; $m=$ Elektronenmasse; $Z_{n}=$ Kernladungszahl des $n$-ten Kerns; $b_{n}$ bzw. $c_{n}$ sind die $b$ - bzw. $c$-Koordinate des $n$-ten Kernes. $I_{a a}^{(n)}$ ist das Trägheitsmoment der Kerne um die $a$-Achse des kerngerüstfesten Koordinatensystems. $L_{a}=\sum_{e}\left(b_{e} p_{c e}-c_{e} p_{b e}\right)=$ Operator für die $a$-Kompo nente des elektronischen Drehimpulses. (Die „eSumme" läuft über sämtliche Elektronen des Moleküls.) Die Störsummen in (1) laufen über die angeregten Elektronenzustände des "fixed center". Problems.

Aus den Beziehungen (1) lassen sich dann die Erwartungswerte für die Elektronenkoordinatenquadrate im Elektronengrundzustand $\left\langle 0\left|\sum_{e} a_{e}{ }^{2}\right| 0\right\rangle$ etc. und die Diagonalelemente des elektrischen Quadrupolmoments des Moleküls berechnen.

1 J. R. Eshbach u. M. W. Strandberg, Phys. Rev. 85, 24 [1952]

2 J. H. VAN VLECK, The Theory of Electric and Magnetic Susceptibilities, Oxford University Press, Amen House, London E.C. 4 (First Edition 1932).

3 C. H. Townes, G. C. Dousmanis, R. L. White u. R. F. Schwarz, Discussion Faraday Soc. 19, 56 [1955]. Mei-Ku Lo, V. W. Weiss u. W. H. Flygare, J. Chem. Phys. 45, 2439 [1966].
Der Vergleich der $g$-Faktoren isotopensubstituierter Moleküle erlaubt die Bestimmung des Vorzeichens des elektrischen Dipolmoments ${ }^{3}$. Die Kenntnis der Diagonalelemente des magnetischen Suszeptibilitätstensors läßt Rückschlüsse auf das Ringstromverhalten der untersuchten Moleküle $\mathrm{zu}^{4}$.

Besonders in jüngster Zeit wurde eine große Zahl von Molekülen mit näherungsweise als starr annehmbarem Kerngerüst [nur für solche gelten die Beziehungen (1)] untersucht ${ }^{5}$.

Für Moleküle mit annähernd frei beweglichen Teilgruppen liegen dagegen unseres Wissens fast keine experimentellen Daten vor ${ }^{6}$.

Insbesondere wurden bisher keine den Beziehungen (1) entsprechende Ausdrücke hergeleitet, die eine Interpretation der gemessenen effektiven $g$ bzw. $\chi$-Werte gestatten und Rückschlüsse auf die Elektronenverteilung des Moleküls erlauben würden.

In der vorliegenden Arbeit soll der HamiltonOperator für Moleküle gegeben werden, die man sich näherungsweise aus einem starren Molekülrumpf und einem auf diesen aufgesteckten symmetrischen um seine Symmetrieachse drehbaren Teilkreisel zusammengesetzt denken kann. (Beispielsweise $\mathrm{CH}_{3}->-\mathrm{F}$ mit der $\mathrm{CH}_{3}$-Gruppe als rumpf.)

${ }^{4}$ D. Sutter u. W. H. Flygare, J. Amer. Chem. Soc. 91, 6895 [1969]. - R. L. Shoemaker u. W. H. Flygare, J. Chem. Phys. 51, 2988 [1969].

5 Eine ausführliche Übersicht über die neueren Arbeiten findet man in dem Ubersichtsartikel von H. D. RUDOLPH, in Annual Reviews of Physical Chemistry, Vol. 21, Annual Reviews Inc., Palo Alto, California, USA [1970] (im Druck).

6 W. Hüttner u. W. H. Flygare, Trans. Faraday Soc. 65, 1953 [1969]. 
Der Teilkreisel soll um seine Symmetrieachse drehbar sein. Die mit der Teilkreiselrotation verknüpften elektrischen Kreisströme verursachen ein zusätzliches magnetisches Moment. In einem äußeren Magnetfeld erwartet man daher eine unterschiedliche Zeeman-Aufspaltung von A-Spezies-Linien (Erwartungswert des Teilkreiseldrehimpulses $p$ gleich Null) und E-Spezies-Linien (Erwartungswert von $p$ ungleich Null) ein und desselben Rotationsüberganges.

Ausgangspunkt unserer Betrachtung ist die Lagrange-Funktion eines Moleküls im Magnetfeld.

$$
\mathcal{L}=(1 / 2) \sum_{n} m_{n} \boldsymbol{v}_{n}^{2}+(1 / c) \sum_{n} q_{n} \boldsymbol{v}_{n} \boldsymbol{A}_{n}-V_{\text {Coulomb }}
$$

$m_{n}=$ Masse, $q_{n}=$ Ladung, $\boldsymbol{v}_{n}=$ Geschwindigkeit, $\boldsymbol{A}_{n}=$ Vektorpotential des äußeren Magnetfeldes am Ort des $n$-ten Teilchens.

In (2) sind alle molekülinternen magnetischen Wechselwirkungen, die in den meisten Fällen zu experimentell (mit einem Mikrowellenspektrometer) nicht auflösbaren Feinheiten der Zeeman-Spektren führen, vernachlässigt.

In Anlehnung an das in der Rotationsspektroskopie übliche Vorgehen führen wir ein kerngerüstfestes Koordinatensystem ein, dessen Ursprung im Schwerpunkt des Kerngerüsts ruht ${ }^{*}$. Weiter wollen wir annehmen, der Kerngerüstschwerpunkt bewege sich nicht relativ zum äußeren Feld. Der Einfluß der thermischen Translationsbewegung im äußeren Feld läßt sich in guter Näherung als Stark-Effekt in einem schwachen elektrischen Feld $\boldsymbol{E}$ beschreiben und ist für Moleküle mit in $M$ und $\boldsymbol{E}$ quadratischem StarkEffekt vernachlässigbar. $(M \hbar=$ Komponente des Moleküldrehimpulses in Richtung der durch die Polarisation der Mikrowellenstrahlung ausgezeichneten raumfesten $Z$-Achse). Bei Rotationsübergängen mit linearem Stark-Effekt kann sich die thermische Translationsbewegung relativ zum Magnetfeld dagegen durchaus in einer Linienverbreiterung bemerkbar machen ${ }^{7}$.

Schon in der ersten experimentellen Arbeit zum Zeeman-Effekt freier Moleküle ${ }^{8}$ zeigte sich, daß

\footnotetext{
* Dadurch wird die Separation von Translation einerseits und Schwingung und Rotation andererseits allerdings aufgehoben. Im folgenden wird die Translation dennoch vernachlässigt, was einer Vernachlässigung der „Mitbewegung des Kerngerüsts" entspricht und in ${ }^{7}$ diskutiert wurde.

7 D. Sutter, A. Guarnieri u. H. Dreizler, Z. Naturforsch. 25 a, 222 [1970]. In dieser Arbeit sind folgende Druckfeh-
}

man zur Interpretation der Zeeman-Feinstruktur die Kopplung von Rotationsbewegung und Elektronenbewegung nicht im Sinne der Born-OppenheimerNäherung vernachlässigen kann. Die bei einer Beschreibung im kerngerüstfesten rotierenden Bezugssystem auftretenden Coriolis-Kräfte mischen in der Sprache der Störungsrechnung der Grundzustandsfunktion der Elektronen ausreichend angeregte Elektronenzustände bei, um ein elektronisches magnetisches Moment der Größenordnung von etwa 1/100 bis 1 Bohrschen Kernmagneton zu erzeugen. Dies kommt in den in Gl. (1) auftretenden Störsummen zum Ausdruck.

Bei Molekülen mit einem annähernd frei drehbaren Teilkreisel erscheint es im Hinblick auf die später durchzuführende Störungsrechnung zweckmäßig, zwei Koordinatensysteme einzuführen.

1. Das Trägheitshauptachsensystem des Kerngerüsts des Gesamtmoleküls (Koordinaten: $a, b, c$; die Lage des Achsensystems ist wegen der vorausgesetzten mindestens dreizähligen Symmetrie des Teilkreisels unabhängig vom Drehwinkel $\alpha$ ).

2. Das Trägheitshauptachsensystem des Kerngerüsts des Teilkreisels (Koordinaten: $\xi, \eta, \zeta$ ).

Beschreibt man dann die „Teilkreiselelektronenfunktionen“ im „, $\xi \eta \zeta$-System“, die „Rumpfelektronenfunktionen “ im „abc-System“, so erreicht man im allgemeinen wohl, daß die Elektronenfunktionen $\Psi$ - in diesen Koordinaten beschrieben - nur schwach von $\alpha$ abhängen bzw. daß man $\partial \Psi / \partial \alpha$ in erster Näherung gleich Null setzen kann. Dies führt zu einer wesentlichen Vereinfachung bei der Störungsrechnung. Implizit ist hierbei vorausgesetzt, daß man die Elektronen- $\Psi$-Funktion in guter Näherung als ein Produkt aus einer Rumpf-Elektronenfunktion und einer Teilkreisel-Elektronenfunktion schreiben kann, d. h. daß die an den Teilkreiselbindungen beteiligten Elektronenorbitale nicht auf den Molekülrumpf übergreifen und umgekehrt. Für Moleküle mit niedrigem Hinderungspotential von nur wenigen cal/Mol glauben wir, daß dies eine vernünftige Annahme ist. Typische Beispiele wären etwa:

ler enthalten: In Gl. (4) muß noch das Glied $-\mathrm{V}^{\prime}$ hinzugefügt werden. Das Vorzeichen des Teils (25 d) muß „-“ sein, nicht wie angegeben „+“. Der zweite Summand in der Klammer von Gl. (17) muß lauten: $J \cdot g^{\mathrm{t}} \cdot \mathrm{H}$.

8 R. Frisch u. O. Stern, Z. Physik 85, 4 [1933]. - G. C. WICk, Z. Physik 85, 25 [1933] und Nuovo Cim. 10, 118 [1933]. 


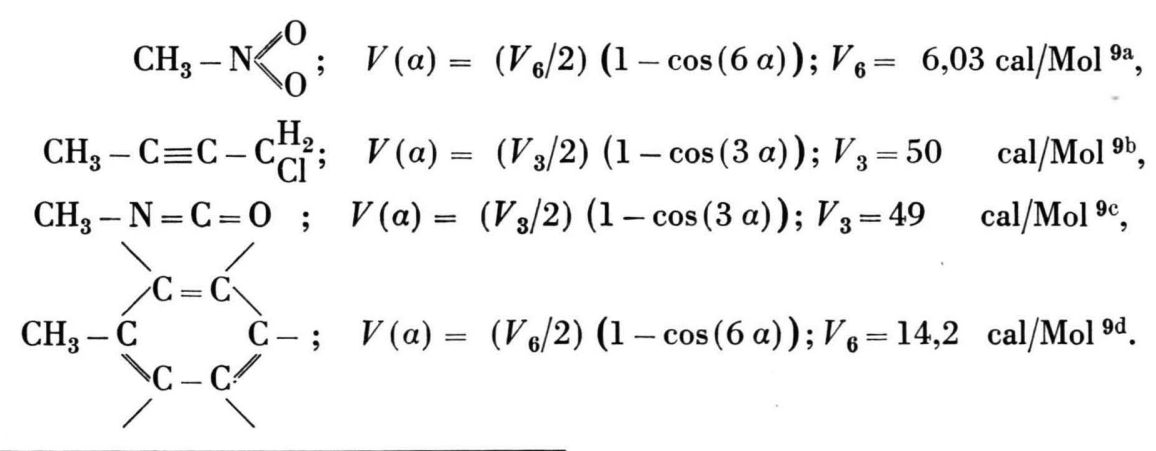

(Eine Reihe weiterer Verbindungen mit fast frei drehbaren Methylgruppen ist in ${ }^{10 b}$, S. $146 \mathrm{ff}$., angegeben.) Die Wahl der beiden Hauptachsensysteme als Bezugssysteme zur Beschreibung der Elektronenorbitale bedeutet im übrigen keinerlei Einschränkung der Gültigkeit der folgenden Betrachtungen und kommt erst bei der Störungsrechnung zum Tragen. Im einzelnen führen wir die folgenden Bezeichnungen ein (vgl. auch Abb. 1) :

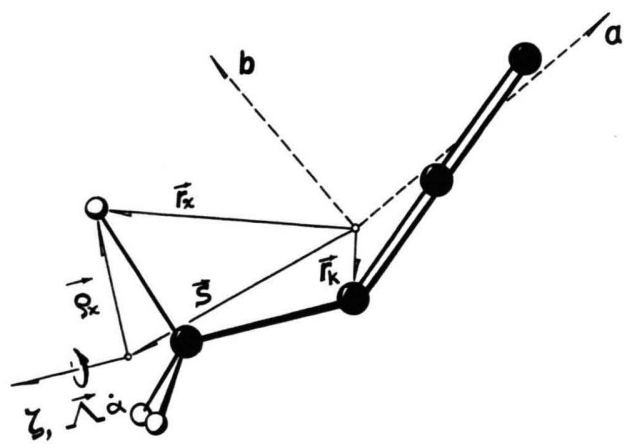

Abb. 1. $\mathrm{CH}_{3} \mathrm{NCO}$ als Beispiel für ein Molekül mit niedrigem Hinderungspotential für die Methylgruppe. Die Rumpfkerne sind schwarz gezeichnet. Die Schwerpunkte des Kerngerüsts des Gesamtmoleküls und des Teilkreiselkerngerüsts sind als kleine Kreise angedeutet. Das Hinderungspotential von $\mathrm{CH}_{3} \mathrm{NCO}$ läßt sich näherungsweise durch $V(a)=\left(V_{3} / 2\right)$ $\cdot(1-\cos (3 \alpha))$ mit $V_{3}=49 \mathrm{cal} / \mathrm{Mol}=0,0021 \mathrm{eV}$ beschreiben.

$\boldsymbol{s}=$ Vektor vom Schwerpunkt des Kerngerüsts des Gesamtmoleküls zum Schwerpunkt des Teilkreiselkerngerüsts,

$\Lambda=$ Einheitsvektor in Richtung der Symmetrie- und Drehachse des Teilkreisels,

9 a: E. R. Tannenbaum, J. Myers u. W. D. Gwinn, J. Chem. Phys. 25, 42 [1956]. b: V. W. Laurie u. D. R. Lide, J. Chem. Phys. 31, 939 [1959]. c: R. F. Curl, V. W. Rao, K. V. L. N. Sastry u. J. A. Hodgeson, J. Chem. Phys. 39, 3335 [1963]. d: H.D. Rudolph, H.DREIZLER, A. JAESCHKe u. P. Wendling, Z. Naturforsch. 22 a, 940 [1967]. $\boldsymbol{r}_{k}=$ Vektor vom Gesamtschwerpunkt zum $k$-ten Kern,

$\boldsymbol{r}_{e}=$ Vektor vom Gesamtschwerpunkt zum $e$-ten Elektron,

$\boldsymbol{\rho}_{x}=$ Vektor vom Schwerpunkt des Teilkreisels zum $\varkappa$-ten Kern des Teilkreisels,

$\boldsymbol{\rho}_{\varepsilon}=$ Vektor vom Schwerpunkt des Teilkreisels zum $\varepsilon$-ten Elektron des Teilkreisels.

Allgemein sollen für „Rumpfteilchen“ lateinische, für Teilkreiselteilchen griechische Indizes verwendet werden. Für den Teilkreisel führen wir zusätzlich ein mit den Teilkreiselkernen fest verbundenes Koordinatensystem $\xi, \eta, \zeta$ ein, dessen Ursprung im Schwerpunkt der Teilkreiselkerne und dessen $\zeta$ Achse in Richtung der Symmetrie und Drehachse des Teilkreiselkerngerüstes liegen soll. Seine Drehlage relativ zum Kerngerüsthauptachsensystem des Gesamtmoleküls soll durch den Drehwinkel $\alpha$ beschrieben werden.

Die in Gl. (2) auftretenden Geschwindigkeiten lassen sich dann wie folgt schreiben:

Rumpfkerne: $\boldsymbol{v}_{k}=\boldsymbol{\omega} \times \boldsymbol{r}_{k}$,

Teilkreiselkerne: $\quad \boldsymbol{v}_{x}=\boldsymbol{\omega} \times \boldsymbol{r}_{\varkappa}+\alpha\left(\boldsymbol{\Lambda} \times \boldsymbol{p}_{x}\right)$,

Rumpfelektronen: $\quad \boldsymbol{v}_{e}=\boldsymbol{\omega} \times \boldsymbol{r}_{e}+\dot{\boldsymbol{r}}_{e}$,

Teilkreiselektronen: $\quad \boldsymbol{v}_{\varepsilon}=\boldsymbol{\omega} \times \boldsymbol{r}_{\varepsilon}+\alpha\left(\boldsymbol{\Lambda} \times \boldsymbol{p}_{\varepsilon}\right)+\dot{\boldsymbol{\rho}}_{\varepsilon}$

$\boldsymbol{\omega}$ ist die momentane Winkelgeschwindigkeit des rotierenden Kerngerüsts (Komponenten: $\omega_{a}, \omega_{b}, \omega_{c}$ ). $\dot{\boldsymbol{r}}_{e}$ ist die Geschwindigkeit des $e$-ten Elektrons relativ zum Hauptachsenkoordinatensystem des Gesamtmoleküls (Komponenten: $\dot{a}_{e}, \dot{b}_{e}, \dot{c}_{e}$ ). $\dot{\boldsymbol{r}}_{\varepsilon}$ ist die Geschwindigkeit des $\varepsilon$-ten Teilkreiselelektrons relativ

10 a) Chun C. Lin u. Jerome D. Swalen, Rev. Mod. Phys. 31, 841 [1959]. - b) H. Dreizler, in: Fortschritte der chemischen Forschung, Vol. 10, Springer-Verlag, BerlinGöttingen-Heidelberg 1968, S. 59. 
$E$

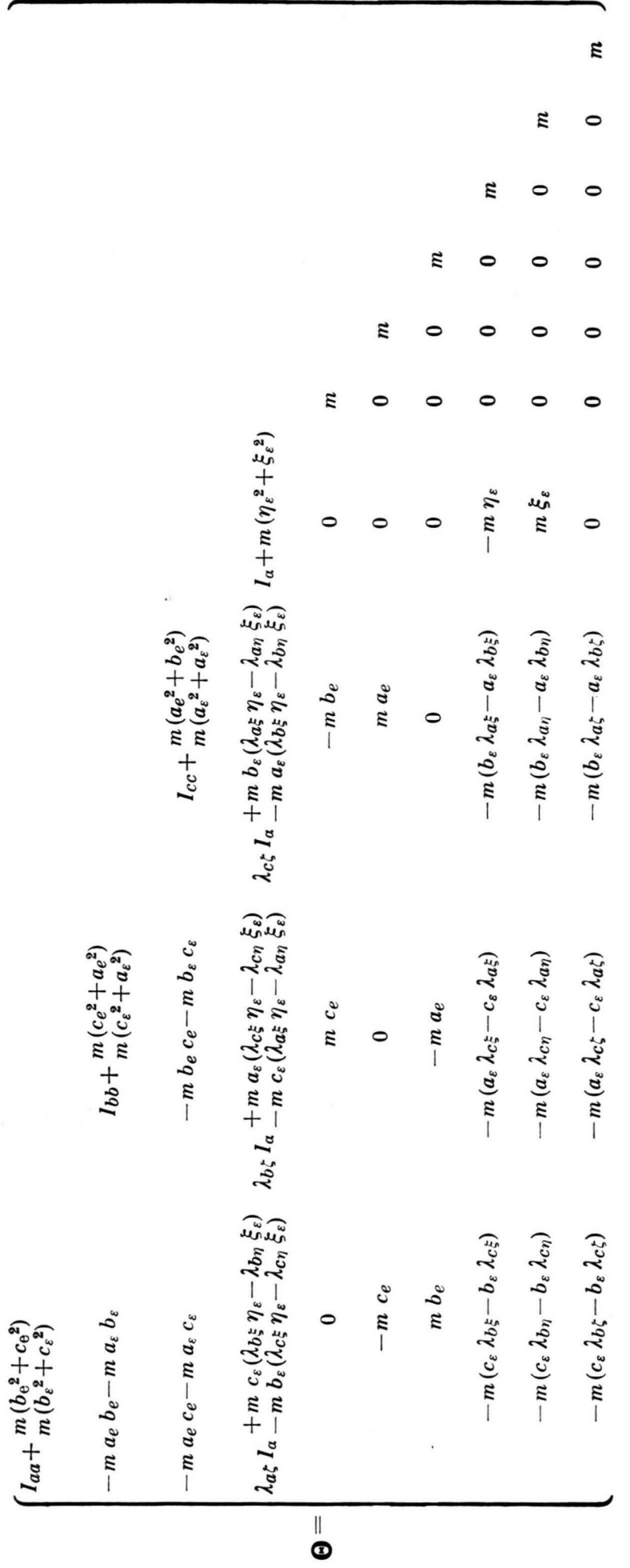

$\frac{a}{\infty}$

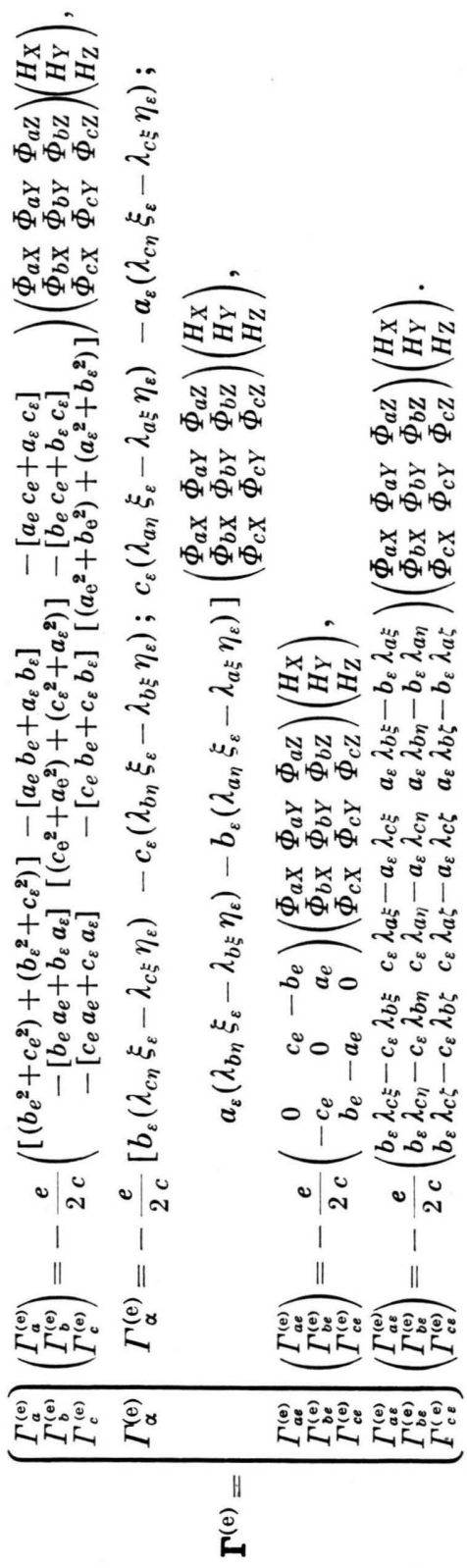


zum Teilkreiselsystem (Komponenten: $\dot{\xi}_{\varepsilon}, \dot{\eta}_{\varepsilon}, \dot{\zeta}_{\varepsilon}$ ).

Für das Vektorpotential des äußeren, als homogen vorausgesetzten, magnetischen Feldes am Ort des $n$-ten Teilchens läßt sich schreiben:

$$
\boldsymbol{A}_{n}=(1 / 2)\left(\boldsymbol{H} \times \boldsymbol{r}_{n}\right) .
$$

Geht man mit (3) und (4) in die Lagrange-Funktion ein, so erhält man einen etwas komplizierten Ausdruck, der sich in Matrizenform übersichtlicher schreiben läßt. Es ergibt sich:

$$
\mathcal{L}=1 / 2 \boldsymbol{\Omega}^{\mathrm{t}} \cdot \boldsymbol{\Theta} \cdot \boldsymbol{\Omega}+\boldsymbol{\Omega}^{\mathrm{t}} \cdot \boldsymbol{\Gamma}-V_{\text {Coulomb }} .
$$

Hierin ist $\boldsymbol{\Omega}$ ein verallgemeinerter Geschwindigkeitsvektor, $\boldsymbol{\Theta}$ eine verallgemeinerte Trägheitsmatrix und $\boldsymbol{\Gamma}$ ein von der magnetischen Feldstärke linear abhängiger "Zeeman-Vektor“. $\boldsymbol{\Omega}^{\mathrm{t}}$ ist die zu $\boldsymbol{\Omega}$ transponierte Matrix.

$$
\boldsymbol{\Omega}^{\mathrm{t}}=\left(\omega_{a}, \omega_{b}, \omega_{c}, \dot{\alpha}, \dot{a}_{e}, \dot{b}_{e}, \dot{c}_{e}, \dot{\xi}_{\varepsilon}, \dot{\eta}_{\varepsilon}, \dot{\zeta}_{\varepsilon}\right),
$$

Die Matrizen haben explizit die in (7) angegebene Gestalt (s. S. 1039). Die $\boldsymbol{\Theta}$-Matrix ist symmetrisch. Nur die untere Hälfte ist explizit angegeben. Darüber hinaus ist jeweils nur 1 Rumpfelektron (Hauptachsenkoordinaten: $a_{e}, b_{e}, c_{e}$ ) und 1 Teilkreiselelektron (Hauptachsenkoordinaten: $a_{\varepsilon}, b_{\varepsilon}, c_{\varepsilon}$; Teilkreiselkoordinaten: $\xi_{\varepsilon}, \eta_{\varepsilon}, \zeta_{\varepsilon}$ ) explizit angeführt.

$$
\begin{aligned}
I_{a a} & =\sum_{n} M_{n}\left(b_{n}{ }^{2}+c_{n}{ }^{2}\right), \\
I_{b b} & =\sum_{n} M_{n}\left(c_{n}{ }^{2}+a_{n}{ }^{2}\right), \\
I_{c c} & =\sum_{n}^{n} M_{n}\left(a_{n}{ }^{2}+b_{n}{ }^{2}\right)
\end{aligned}
$$

sind die Hauptträgheitsmomente des Gesamtkerngerüsts. Die Summen laufen über sämtliche Kerne des Moleküls und sind wegen der Symmetrie des Teilkreisels um seine Drehachse unabhängig vom Drehwinkel $\alpha . M_{n}$ ist die Masse des $n$-ten Kernes. $I_{\alpha}=\sum_{\varkappa} M_{x}\left(\xi_{x}{ }^{2}+\eta_{x}{ }^{2}\right)$ ist das Trägheitsmoment der Teilkreiselkerne um dessen Symmetrie- und DrehAchse. $\lambda_{a \xi}$ ist der Richtungscosinus zwischen der molekülfesten $a$-Achse und der teilkreiselfesten $\xi$ Achse (entsprechend für die übrigen Achspaarungen). Da die Drehachse mit der $\zeta$-Achse zusammenfällt, sind $\lambda_{a \zeta}, \lambda_{b \zeta}$ und $\lambda_{c \zeta}$ von $\alpha$ unabhängig, während die Richtungscosinus zwischen den Hauptachsen und der $\xi$ - bzw. $\eta$-Achse des Teilkreisels Funktionen von $\alpha$ sind.

Der „Zeeman-Vektor" $\boldsymbol{\Gamma}$ läßt sich schreiben als Summe eines „Kern-Zeeman-Vektors" $\Gamma^{(n)}$ und eines „Elektronen-Zeeman-Vektors“ $\boldsymbol{\Gamma}^{(\mathrm{e})}$. Vom Kern-Zeeman-Vektor sind nur die ersten vier Komponenten von Null verschieden. Sie lauten:

$$
\begin{aligned}
\left(\begin{array}{l}
\Gamma_{a}^{(\mathrm{n})} \\
\Gamma_{b}^{(\mathrm{n})} \\
\Gamma_{c}^{(\mathrm{n})}
\end{array}\right) & =\frac{e}{2 c} \sum_{n}\left(\begin{array}{ccc}
Z_{n}\left(b_{n}^{2}+c_{n}^{2}\right) & -Z_{n} a_{n} b_{n} & -Z_{n} a_{n} c_{n} \\
-Z_{n} b_{n} a_{n} & Z_{n}\left(c_{n}^{2}+a_{n}^{2}\right) & -Z_{n} b_{n} c_{n} \\
-Z_{n} c_{n} a_{n} & -Z_{n} c_{n} b_{n} & Z_{n}\left(a_{n}^{2}+b_{n}^{2}\right)
\end{array}\right)\left(\begin{array}{ccc}
\Phi_{a X} & \Phi_{a Y} & \Phi_{a Z} \\
\Phi_{b X} & \Phi_{b Y} & \Phi_{b Z} \\
\Phi_{c X} & \Phi_{c Y} & \Phi_{c Z}
\end{array}\right)\left(\begin{array}{l}
H_{X} \\
H_{Y} \\
\mathrm{H}_{Z}
\end{array}\right) \\
\Gamma_{\alpha}^{(\mathrm{n})} & =\frac{e}{2 c} \cdot \frac{Z_{T}}{M_{T}} \cdot I_{\alpha}\left(\lambda_{a \zeta}, \lambda_{b \zeta}, \lambda_{c \zeta}\right)\left(\begin{array}{lll}
\Phi_{a X} & \Phi_{a Y} & \Phi_{a Z} \\
\Phi_{b X} & \Phi_{b Y} & \Phi_{b Z} \\
\Phi_{c X} & \Phi_{c Y} & \Phi_{c Z}
\end{array}\right)\left(\begin{array}{c}
H_{X} \\
H_{Y} \\
H_{Z}
\end{array}\right) .
\end{aligned}
$$

Hierin ist $Z_{n}$ die Kernladungszahl des $n$-ten Atomkerns. Die $n$-Summation läuft wieder über sämtliche Atomkerne des Moleküls und ist, wenn der Teilkreisel nicht nur massensymmetrisch, sondern auch ladungssymmetrisch ist, von $\alpha$ unabhängig.

$e \quad$ ist der Betrag der elektrischen Elementarladung, $c$ die Lichtgeschwindigkeit.

$\Phi_{a X}$ ist der Richtungscosinus zwischen der kerngerüstfreien $a$-Achse und der raumfesten $X$-Achse (entsprechend die übrigen Achspaarungen).

$H_{X}$ ist die Komponente des Magnetfeldes in der raumfesten $X$-Richtung etc.

Im Ausdruck für $\Gamma_{\alpha}^{(\mathrm{n})}$ ist schon auf einen Teilkreisel, der aus identischen Atomen aufgebaut ist, spezialisiert. $Z_{T}$ ist die Kernladungszahl dieser Atome, $M_{T}$ deren Masse.

Der elektronische „Zeeman-Vektor“ ist in (8 b) angegeben (s. S. 1039).
Im nächsten Schritt führen wir die zu den Geschwindigkeiten konjugierten Impulse ein:

$$
\begin{aligned}
P_{a}=\partial \mathcal{L} / \partial \omega_{a}, & p_{a}=\partial \mathcal{L} / \partial \dot{\alpha}, \\
& p_{a e}=\partial \mathcal{L} / \partial \dot{a}_{e}, p_{\xi \varepsilon}=\partial \mathcal{L} / \partial \dot{\xi}_{\varepsilon} \quad \text { usw. }
\end{aligned}
$$

Aus (5) folgt für den Impuls-Spaltenvektor $\boldsymbol{P}$ :

$$
\boldsymbol{P}=\boldsymbol{\Theta} \cdot \boldsymbol{\Omega}+\boldsymbol{\Gamma} \text { bzw. } \boldsymbol{\Omega}=\boldsymbol{\Theta}^{-1} \cdot(\boldsymbol{P}-\boldsymbol{\Gamma}) .
$$

Für die Hamilton-Funktion ergibt sich damit:

$$
\begin{aligned}
\mathcal{H}= & \boldsymbol{\Omega}^{\mathrm{t}} \cdot \boldsymbol{P}-\mathcal{L}=(1 / 2)(\boldsymbol{P}-\boldsymbol{\Gamma})^{\mathrm{t}} . \\
& \cdot \boldsymbol{\Theta}^{-1} \cdot(\boldsymbol{P}-\boldsymbol{\Gamma})+V_{\text {Coulomb }} .
\end{aligned}
$$

Die in (10) notwendige Inversion von $\boldsymbol{\Theta}$ wird wesätlich vereinfacht, wenn man zunächst in (5) durch eine Vortransformation $\boldsymbol{T}$ zu neuen Geschwindigkeiten übergeht ${ }^{7}$. Mit

$$
\boldsymbol{\Omega}=\boldsymbol{T} \cdot \widetilde{\boldsymbol{\Omega}}
$$




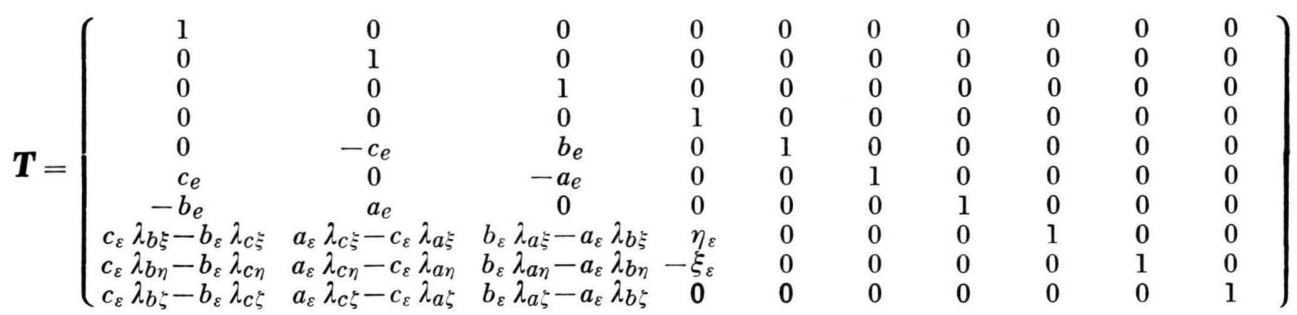

geht (5) über in:

$$
\mathcal{L}=(1 / 2) \widetilde{\boldsymbol{\Omega}}^{\mathrm{t}} \cdot \widetilde{\boldsymbol{\Theta}} \cdot \widetilde{\boldsymbol{\Omega}}+\widetilde{\boldsymbol{\Omega}}^{\mathrm{t}} \cdot \widetilde{\boldsymbol{\Gamma}}-V_{\text {Coulomb }}
$$

mit

$$
\begin{aligned}
\widetilde{\boldsymbol{\Theta}} & =\boldsymbol{T}^{\mathrm{t}} \cdot \boldsymbol{\Theta} \cdot \boldsymbol{T}, \\
\widetilde{\boldsymbol{\Gamma}} & =\boldsymbol{T}^{\mathrm{t}} \cdot \boldsymbol{\Gamma} .
\end{aligned}
$$

Hierin hat die neue „Trägheitsmatrix“ $\widetilde{\Theta}$ die wesentlich einfachere Form:

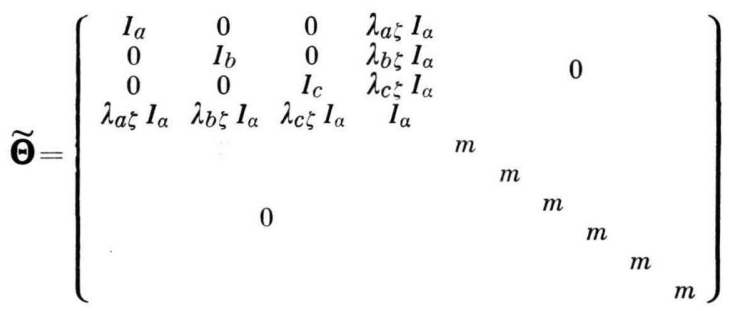

Die vier auf vier Submatrix in der linken oberen Ecke von (15) hat die vom Einkreiselproblem der internen Rotation her wohlbekannte Gestalt ${ }^{10}$. Sämtliche Matrixelemente von $\widetilde{\boldsymbol{\Theta}}$ sind für das betreffende Molekül konstant, da wir Starrheit von Rumpf- und Teilkreisel-Kerngerüst vorausgesetzt haben.

Auch der neue „Zeeman-Vektor“ $\widetilde{\mathbf{\Gamma}}$ ist wesentlich vereinfacht. In den ersten vier Komponenten ist nur noch der Kernanteil von $\tilde{\mathbf{I}}$ übriggeblieben. Die restlichen „rein elektronischen“ Komponenten werden durch die Transformation dagegen nicht beeinflußt.

Analog zu (10) ergibt sich als neue HamiltonFunktion:

$$
\mathcal{H}=(1 / 2)(\widetilde{\boldsymbol{P}}-\widetilde{\mathbf{\Gamma}})^{\mathrm{t}} \cdot \widetilde{\boldsymbol{\Theta}}^{-1} \cdot(\widetilde{\boldsymbol{P}}-\widetilde{\mathbf{\Gamma}})+V_{\text {Coulomb }} \cdot
$$

Vor dem Übergang zur Quantenmechanik gehen wir wieder zurück zu den ursprünglichen Impulsen $\boldsymbol{P}$.
Mit

erhält man:

$$
\widetilde{\boldsymbol{P}}=\boldsymbol{T}^{\mathrm{t}} \boldsymbol{P}
$$

$$
\widetilde{P}_{a}=P_{a}-L_{a}^{\mathrm{R}}-L_{a}^{\mathrm{T}} \quad \text { (und zyklisch). }
$$

Hierin sind $L_{a}^{\mathrm{R}}$ und $L_{a}^{\mathrm{T}}$ elektronische Anteile des Drehimpulses um die $a$-Achse:

$$
L_{a}^{\mathbf{R}}=\sum_{e}\left(b_{e} p_{c e}-c_{e} p_{b e}\right) .
$$

(Die Summe läuft nur über die „Rumpf-Elektronen“.)

$$
\begin{aligned}
L_{a}^{\mathrm{T}}=\sum_{\varepsilon}\left(b_{\varepsilon}\left(\lambda_{c \xi} p_{\xi \varepsilon}+\lambda_{c \eta} p_{\eta \varepsilon}+\lambda_{c \zeta} p_{\zeta \varepsilon}\right)\right. & \\
& \left.-c_{\varepsilon}\left(\lambda_{b \xi} p_{\xi \varepsilon}+\lambda_{b \eta} p_{\eta \varepsilon}-\lambda_{b \xi} p_{\zeta \varepsilon}\right)\right) .
\end{aligned}
$$

(Die Summe läuft nur über die Teilkreisel-Elektronen.)

Für die vierte Komponente von $\boldsymbol{P}$ erhält man:

$$
\tilde{p}_{\alpha}=p_{\alpha}-L_{\alpha}^{\mathrm{T}}
$$

mit

$$
L_{\alpha}^{\mathrm{T}}=\sum_{\varepsilon}\left(\xi_{\varepsilon} p_{\eta \varepsilon}-\eta_{\varepsilon} p_{\xi \varepsilon}\right) .
$$

Die rein elektronischen Anteile von $\widetilde{\boldsymbol{P}}$ stimmen mit denen von $\boldsymbol{P}$ überein, d. h.

$$
\begin{array}{cl}
\widetilde{p}_{a e}=p_{a e} & \text { etc. } \\
\tilde{p}_{\xi \varepsilon}=p_{\xi \varepsilon} & \text { etc. }
\end{array}
$$

Denkt man sich für einen Augenblick das Magnetfeld abgeschaltet, so erkennt man [vgl. Gln. (7), (9) und (18) ], daß dann $\widetilde{P}_{a}$ gerade der vom Kerngerüst des Gesamtmoleküls stammende Drehimpuls um die $a$-Achse (und zyklisch) und $p_{a}$ der von $\alpha$ und $\omega$-Rotation herrührende Drehimpuls der Teilkreiselkerne um eine im betreffenden Augenblick mit der Teilkreiselachse zusammenfallende raumfeste Drehachse sind.

Nach Einsetzen des Ausdrucks (18) für $\boldsymbol{P}$ in die Hamilton-Funktion (16) erhält man diese in ihrer endgültigen Form:

$$
\mathcal{H}=\frac{1}{2 m} \sum_{e}\left(p_{a_{e}}^{2}+p_{b_{e}}^{2}+p_{c_{e}}^{2}\right)+\frac{1}{2 m} \sum_{\varepsilon}\left(p_{\xi_{\varepsilon}}^{2}+p_{\eta_{\varepsilon}}^{2}+p_{\zeta_{\varepsilon}}^{2}\right)+V_{\text {Coulomb }}
$$




$$
\begin{aligned}
& +\frac{1}{2}\left(P_{a}-L_{a}, P_{b}-L_{b}, \quad P_{c}-L_{c}, p_{a}-L_{a}\right)\left(\mathbf{I}^{-1}\right)\left(\begin{array}{c}
P_{a}-L_{a} \\
P_{b}-L_{b} \\
P_{c}-L_{c} \\
p_{a}-L_{a}
\end{array}\right) \\
& -\frac{1}{2}\left\{\left(\Gamma_{a}^{(\mathrm{n})}, \Gamma_{b}^{(\mathrm{n})}, \Gamma_{c}^{(\mathrm{n})}, \Gamma_{\alpha}^{(\mathrm{n})}\right)\left(\boldsymbol{I}^{-1}\right)\left(\begin{array}{c}
P_{a}-L_{a} \\
P_{b}-L_{b} \\
P_{c}-L_{c} \\
p_{\alpha}-L_{\alpha}
\end{array}\right)+\left(P_{a}-L_{a}, P_{b}-L_{b}, P_{c}-L_{c}, p_{a}-L_{\alpha}\right)\left(I^{-1}\right)\left(\begin{array}{c}
T_{a}^{(\mathrm{n})} \\
\Gamma_{b}^{(\mathrm{n})} \\
\Gamma_{c}^{(\mathrm{n})} \\
\Gamma_{\alpha}^{(\mathrm{n})}
\end{array}\right)\right\} \\
& +\frac{1}{2}\left(\Gamma_{a}^{(\mathrm{n})}, \Gamma_{b}^{(\mathrm{n})}, \Gamma_{c}^{(\mathrm{n})}, \Gamma_{\alpha}^{(\mathrm{n})}\right)\left(\boldsymbol{I}^{-1}\right)\left(\begin{array}{c}
\Gamma_{a}^{(\mathrm{n})} \\
\Gamma_{b}^{(\mathrm{n})} \\
\Gamma_{c}^{(\mathrm{u})} \\
\Gamma_{\alpha}^{(\mathrm{n}}
\end{array}\right) \\
& +\frac{e}{2 m c}\left(L_{a}, L_{b}, L_{c}\right)\left(\begin{array}{lll}
\Phi_{a X} & \Phi_{a Y} & \Phi_{a Z} \\
\Phi_{b X} & \Phi_{b Y} & \Phi_{b Z} \\
\Phi_{c X} & \Phi_{c Y} & \Phi_{c Z}
\end{array}\right)\left(\begin{array}{c}
H_{X} \\
H_{Y} \\
H_{Z}
\end{array}\right) \\
& +\frac{e^{2}}{8 m c}\left(H_{X}, H_{Y}, H_{Z}\right)\left(\begin{array}{lll}
\Phi_{a X} & \Phi_{b X} & \Phi_{c X} \\
\Phi_{a Y} & \Phi_{b Y} & \Phi_{c Y} \\
\Phi_{a Z} & \Phi_{b Z} & \Phi_{c Z}
\end{array}\right)\left(\begin{array}{ccc}
\sum_{j}\left(b_{j}{ }^{2}+c_{j}{ }^{2}\right) & -\sum_{j} a_{j} b_{j} & -\sum a_{j} c_{j} \\
-\sum_{j} b_{j} a_{j} & \sum_{j}\left(c_{j}^{2}+a_{j}{ }^{2}\right) & -\Sigma b_{j} c_{j} \\
-\sum c_{j} a_{j} & -\sum c_{j} b_{j} & \sum_{j}\left(a_{j}{ }^{2}+b_{j}{ }^{2}\right)
\end{array}\right)\left(\begin{array}{lll}
\Phi_{a X} & \Phi_{a Y} & \Phi_{a Z} \\
\Phi_{b X} & \Phi_{b Y} & \Phi_{b Z} \\
\Phi_{c X} & \Phi_{c Y} & \Phi_{c Z}
\end{array}\right)\left(\begin{array}{c}
H_{X} \\
H_{Y} \\
H_{Z}
\end{array}\right) \text {. }
\end{aligned}
$$

Hierin ist

$$
I^{-1}=\left(\begin{array}{cccc}
\left(\frac{1}{I_{a}}+\frac{\lambda^{2} a \zeta I_{\alpha}}{I_{a}{ }^{2} r}\right) & +\frac{\lambda_{a \zeta} \lambda_{b \zeta} I_{\alpha}}{I_{a} I_{b} r} & +\frac{\lambda_{a \zeta} \lambda_{b \zeta} I_{\alpha}}{I_{a} I_{b} r} & -\frac{\lambda_{a \zeta}}{I_{a} r} \\
+\frac{\lambda_{a \zeta} \lambda_{c \zeta} I_{\alpha}}{I_{a} I_{c} r} & \left(\frac{1}{I_{b}}+\frac{\lambda^{2} b \zeta I_{\alpha}}{I_{b}{ }^{2} r}\right) & +\frac{\lambda_{a \zeta} \lambda_{c \zeta} I_{\alpha}}{I_{a} I_{b} r} & -\frac{\lambda_{b \zeta}}{I_{b} r} \\
+\frac{\lambda_{b \zeta} \lambda_{c \zeta} I_{\alpha}}{I_{b} I_{c} r} & +\frac{\lambda_{b \zeta} \lambda_{c \zeta} I_{\alpha}}{I_{b} I_{c} r}\left(\frac{1}{I_{c}}+\frac{\lambda_{c \zeta}^{2} I_{\alpha}}{I_{c}{ }^{2} r}\right) & -\frac{\lambda_{c \zeta}}{I_{c} r} \\
-\frac{\lambda_{a \zeta}}{I_{a} r} & -\frac{\lambda_{b \zeta}}{I_{b} r} & -\frac{\lambda_{c \zeta}}{I_{c} r} & -\frac{1}{I_{\alpha} r}
\end{array}\right)
$$

mit $\quad r=\left(1-\sum_{g} \frac{\lambda_{g \zeta} \boldsymbol{I}_{\alpha}}{\boldsymbol{I}_{g}^{(\mathrm{n})}}\right)$

$$
L_{a}=L_{a}^{(\mathrm{R})}+L_{a}^{(\mathrm{T})}=\sum_{e}\left(b_{e} p_{c e}-c_{e} p_{b e}\right)+\sum_{\varepsilon}\left(b_{\varepsilon}\left(\lambda_{c \xi} p_{\xi \varepsilon}+\lambda_{c \varepsilon} p_{\eta \varepsilon}+\lambda_{c \zeta} p_{\zeta \varepsilon}\right)-c_{\varepsilon}\left(\lambda_{b \xi} p_{\xi \varepsilon}-\lambda_{b \eta} p_{\eta \varepsilon}-\lambda_{b \xi} p_{\zeta \varepsilon}\right)\right) .
$$

(19 a) ist für einen gegebenen Teilkreiselwinkel $\alpha$ die Schrödinger-Gleichung für das "fixed center"Problem der Elektronen.

(19 b) ist formal [vgl. die aus (7) und (9) folgende Bedeutung von $P_{a}$ ] die kinetische Energie des Kerngerüstes.

(19 c) liefert im wesentlichen die Kerngerüst-g-Faktoren (vgl. auch Fußnote S. 227 in ${ }^{7}$ ).

(19 d) liefert den Kernbeitrag zur Suszeptibilität, ähnlich wie im Fall des vollkommen starren Kreisels.

(19 e) liefert Beiträge zu den Elektronen-g-Faktoren und zur Suszeptibilität.

(19 f) liefert einen Elektronen-Beitrag zur Suszeptibilität.

Beim Übergang zur Quantenmechanik sind die Impulse durch die entsprechenden Differentialoperatoren zu ersetzen:

$p_{a e}=\frac{\hbar}{i} \frac{\partial}{\partial a_{e}}$ etc. $\quad p_{\xi \varepsilon}=\frac{\hbar}{i} \frac{\partial}{\partial \xi_{\varepsilon}}$ etc. $\quad p_{\alpha}=\frac{\hbar}{i} \frac{\partial}{\partial \alpha}$.
Für $P_{a}$ ist der Operator zur $a$-Komponente des Drehimpulses zu setzen ausgedrückt in den die momentane Lage des Kerngerüstkoordinatensystems beschreibenden Euler-Winkeln.

Die weitere Behandlung von (19) im Rahmen einer Störungsrechnung, bei der von dem Basisfunktionssystem

$\Psi_{m ; J K M ; v}=\exp (i m \alpha) \cdot \Phi_{J K M}(\varphi, \vartheta, \chi) \cdot \varphi_{v}\left(\boldsymbol{r}_{e}, \rho_{\varepsilon}, \alpha\right)$

ausgegangen wird, soll in einer zweiten Arbeit gegeben werden.

In (22) sind $\exp (i m \alpha)$ die Eigenfunktionen des freien internen Rotators. Die $\Psi_{J K M}(\varphi, \vartheta, \chi)$ sind die Eigenfunktionen des symmetrischen Kreisels in Abhängigkeit von den Euler-Winkeln $\varphi, \vartheta$ und $\gamma$. $\varphi_{v}\left(r_{e}, \varrho_{\varepsilon}, \alpha\right)$ sind die Elektroneneigenfunktionen des "fixed center"-Problems, die "parametrisch" 
vom Drehwinkel $a$ des Teilkreisels relativ zum Rumpf abhängen ( $v$ soll dabei für die Gesamtheit der elektronischen Quantenzahlen stehen).

Vernachlässigt man in (19) sämtliche ElektronenTerme, rechnet also mit einem Modell starrer Punktladungen, so sollte man aus (19 c) schon ohne jede Störungsrechnung die Struktur des endgültigen , $g$ Tensors" für den Fall der internen Rotation erraten können. Einsetzen der Ausdrücke (8 a) für die Punktladungsanteile des „Zeeman-Vektors“ ergibt dabei einen Ausdruck, der dem von HütTner und FLYGARE in ${ }^{6}$ angegebenen Operator entspricht, aber eine Reihe zusätzlicher Glieder enthält, deren Einfluß bei niedrigem Hinderungspotential schwer abzuschätzen ist. Da es sich bei dem in ${ }^{6}$ untersuchten Molekül - Acetaldehyd - um eine Verbindung mit einem vergleichsweise hohen Hinderungspotential $\left(V_{3}=\right.$ $1167 \mathrm{cal} / \mathrm{Mol}$ ) handelt, bei der sich die interne Rotation noch nicht in einem Unterschied der ZeemanAufspaltung von A- und E-Spezies des Torsionsgrundzustands bemerkbar macht, wird die Auswertung der Acetaldehyddaten aber vermutlich von den Zusatzgliedern nicht beeinflußt.

Wir danken Herrn Prof. Dr. H. Dreizler für viele anregende Diskussionen. Der Deutschen Forschungsgemeinschaft sei für die Bereitstellung von Personalmitteln gedankt.

\title{
Low Energy Gamma Transitions in ${ }^{171} \mathrm{Tm}$
}

\author{
M. S. EL-NESR * \\ Nuclear Physics Department, Atomic Energy Establishment, Cairo, U.A.R. \\ (Z. Naturforsch. 25 a, 1043-1045 [1970]; received 21 April 1970)
}

\begin{abstract}
The low energy gamma-ray spectrum of ${ }^{171} \mathrm{Er}$ has been studied with the use of both $\mathrm{Ge}(\mathrm{Li})$ gamma-ray and a high resolution iron free double focusing beta-ray spectrometer. The data provide K-conversion coefficients and multipolarities of eight gamma-rays. Spin and parity assignments $7 / 2+, 3 / 2+, 5 / 2+$ and $5 / 2+$ are given to the $636,676,738$ and $913 \mathrm{keV}$ levels respectively.
\end{abstract}

\section{Introduction}

The decay properties of ${ }^{171} \mathrm{Er}$ were studied earlier by several groups ${ }^{1-5}$, using different techniques. Recently, we have investigated ${ }^{1}$ the decay scheme of ${ }^{171} \mathrm{Er}$ by means of an electron-gamma coincidence spectrometer and the existence of 32 transitions in ${ }^{171} \mathrm{Tm}$ was confirmed. In spite of the extensive studies of $\gamma$-transitions following the decay of ${ }^{171} \mathrm{Er}$, the spin and parity assignments for most of the levels are questionable. Therefore, further studies are necessary in order to draw more definite conclusions about level properties.

The present study was undertaken to determine the internal conversion coefficients and multipolarities of some $\gamma$-rays from which information on excited states of ${ }^{171} \mathrm{Tm}$ could be deduced.

* Present address: Department of Physics, Faculty of Engineering, P.O. Box 1098, Tripoli, Libya.

1 M. S. El-Nesr and M. R. El-Aassar, Z. Phys. 189, 138 [1966].

2 B. E. Raeside, J. J. Reidy, and M. L. Wiedenbeck, Bull. Amer. Phys. Soc. 12, 36 [1966].

\section{Experimental Procedures}

The study of the internal conversion electron spectrum was carried out using a high resolution iron-free double focusing $\beta$-ray spectrometer ${ }^{6}\left(\varrho_{0}=50 \mathrm{~cm}\right)$. With this instrument relative momentum measurements could be made with an accuracy of a few parts in $10^{5}$. With a $(0.2 \times 2) \mathrm{cm}^{2}$ source and a $2 \mathrm{~mm}$ detector slit, a resolution of $\sim 0.15 \%$ is obtained. The detector employed in the present studies was a G.M. counter with $\sim 2 \mathrm{mg}$ $/ \mathrm{cm}^{2}$ mica end window.

The gamma-ray intensity data were recorded using a $2.5 \mathrm{~cm}^{3} \mathrm{Ge}(\mathrm{Li})$ detector having a system resolution (FWHM) of $2.5 \mathrm{keV}$ for the $661 \mathrm{keV} \gamma$-ray of ${ }^{137} \mathrm{Cs}$. The detector was connected through low noise electronics to a 400 channel pulse-height analyser. The experimentally deduced relative efficiency correction curve for the germanium detector is estimated to be accurate to $\pm 3-5 \%$. The energy calibration was carried out before and after each irradiation by using standard sources of well-known energies.

3 J. S. Geiger and R. L. Graham, Chalk River Progress Report AECL-2752 [1967].

4 D. G. Megli, G. P. Agin, V. R. Potnis, and C. E. Mandeville, Nucl. Phys. A 107, 217 [1968].

5 D. E. Raeside, J. J. Reidy, and M. L. Wiedenbeck, Nucl. Phys. A 114, 529 [1968].

6 M. S. EL-NesR and G. M. EL-SAYAD, Int. Rep. 2 UAR, A. E. E. [1965]. 